\title{
Desempenho no Teste de Detecção de Intervalo Aleatório - Random Gap Detection Test (RGDT): estudo comparativo entre mulheres jovens e idosas
}

\author{
Performance in the Random Gap Detection Test (RGDT): \\ comparative study between young and elderly women
}

\author{
Daniela Soares de Queiroz ${ }^{1}$, Fátima Cristina Alves Branco-Barreiro², Teresa Maria Momensohn-Santos ${ }^{3}$
}

\begin{abstract}
RESUMO
Objetivo: Comparar o desempenho entre mulheres jovens e idosas para o Random Gap Detection Test (RGDT). Métodos: Foram avaliadas 72 mulheres, distribuídas em dois grupos: Grupo Controle (GC), composto por 48 jovens (idade média: 23,8 anos) com audição periférica normal, e Grupo Experimental (GE), composto por 24 idosas (idade média 66,8 anos) com audição periférica normal ou curva audiométrica do tipo neurossensorial de grau leve. Os indivíduos dos dois grupos foram submetidos ao RGDT para tons puros, nas frequências de 500, 1000, 2000 e 4000 Hz. Resultados: Todos os indivíduos do GC identificaram intervalo até $40 \mathrm{~ms}$ em todas as frequências, sendo o intervalo médio de respostas entre as frequências 12,2 ms. Com relação ao GE, observamos que 20 dos 24 indivíduos não identificaram o intervalo até $40 \mathrm{~ms}$ em uma ou mais frequiências. A análise dos dados mostrou diferença estatisticamente significante entre o desempenho dos dois grupos $(\mathrm{p}<0,001)$. Conclusões: $\mathrm{O}$ avanço da idade mostrou ser fator de piora no desempenho do RGDT. Este teste mostrou ser um instrumento útil para avaliar os efeitos do envelhecimento na habilidade de resolução temporal auditiva.
\end{abstract}

Descritores: Idoso; Envelhecimento; Audição; Transtornos da audição; Percepção auditiva; Transtornos da percepção auditiva; Testes auditivos; Doenças auditivas centrais

\section{INTRODUÇÃO}

O interesse na relação entre o envelhecimento e o processamento auditivo temporal tem sido crescente nos últimos anos, em vista da frequência com que idosos se queixam de dificuldades para compreender a fala, mesmo sem a presença de perda auditiva periférica ${ }^{(1)}$. Essas queixas podem ser explicadas pelo fato de que o envelhecimento do sistema auditivo não se restringe apenas à sua porção periférica. Embora existam evidências sugerindo degeneração coclear, acredita-se que

Pesquisa realizada no Instituto de Estudos Avançados da Audição - IEAA São Paulo (SP), Brasil.

(1) Mestre, Professora do Instituto de Estudos Avançados da Audição - IEAA - São Paulo (SP), Brasil.

(2) Doutora, Professora da Universidade Bandeirante de São Paulo - UNIBAN - São Paulo (SP), Brasil; e do Instituto de Estudos Avançados da Audição IEAA - São Paulo (SP), Brasil.

(3) Doutora, Professora da Pontifícia Universidade Católica de São Paulo PUC-SP - São Paulo (SP), Brasil; Diretora Clínica e de Pesquisa do Instituto de Estudos Avançados da Audição - IEAA - São Paulo (SP), Brasil.

Endereço para correspondência: Daniela Soares de Queiroz. R. José Ferreira de Castro, 259/22A, Vila Amália, São Paulo (SP), Brasil, CEP: 02615-010. E-mail: queiroz3107@yahoo.com.br

Recebido em: 11/11/2008; Aceito em: 22/3/2009 também ocorra disfunção do sistema nervoso auditivo central, ou seja, que a diminuição no número de células íntegras disponíveis acomete desde as células ciliadas do Órgão de Córti até as células nervosas do lobo temporal, passando por toda a via auditiva nervosa. Estas alterações afetariam a integridade funcional de todo o sistema envolvido no processamento dos estímulos sonoros. O envelhecimento, portanto, não representaria somente a perda da sensibilidade auditiva, mas também a diminuição da eficiência de todas as habilidades auditivas ${ }^{(2)}$. Alterações do processamento auditivo podem ser decorrentes de disfunções morfológicas do sistema nervoso auditivo. Dessa forma, se o envelhecimento é um processo degenerativo que provoca alteração morfológica nas células, estas alterações podem comprometer todas as funções auditivas. Por conta disso, há casos em que o idoso apresenta limiares auditivos dentro dos padrões de normalidade e, mesmo assim, apresenta queixas de compreensão de fala e comunicação.

A integridade dos aspectos temporais da audição é prérequisito para que o sistema auditivo determine a duração dos sons, o tempo de intervalo entre eles e sua ordenação temporal, habilidades essas, essenciais para o processamento auditivo da fala e da música ${ }^{(3)}$. A resolução temporal auditiva, habilidade para perceber ou discriminar como eventos sepa- 
rados segmentos de som, que estão espacialmente próximos no tempo, é necessária para que o indivíduo consiga distinguir a ocorrência de dois estímulos ao invés de um. Transtornos desta habilidade auditiva estão frequentemente relacionados a dificuldades de processamento fonológico e discriminação auditiva de pistas temporais da fala ${ }^{(4)}$.

A capacidade de resolução temporal é responsável pela compreensão da fala contínua e de seus segmentos isolados. Transtornos da capacidade de resolução temporal podem resultar em dificuldades para identificar pequenas variações acústicas da fala e, consequentemente, dificuldade em produzir de forma correta os sons da fala ou em interpretar a mensagem ouvida. A identificação do ponto e modo articulatórios dos sons da fala depende da identificação das transições de formantes, que têm duração muito pequena e que variam de acordo com a velocidade de articulação do falante; assim sendo, quando essa transição for menor que o tempo de resolução do sistema auditivo do ouvinte, ele terá dificuldade para discriminar corretamente os sons da fala ${ }^{(5)}$. Isso explicaria algumas das queixas de compreensão de fala trazidas pelos idosos. Desta forma, quando o idoso refere ouvir, mas não entender, há possibilidade de que a habilidade de resolução temporal deteriorada pelo envelhecimento esteja contribuindo para a dificuldade de compreensão de fala, associada ou não ao prejuízo da função auditiva periférica.

Os estudos sobre a habilidade de resolução temporal foram iniciados na década de 1970, com o desenvolvimento do primeiro teste para avaliar essa habilidade, chamado Wichita Auditory Fusion Test (WAFT). Porém, nessa época, este teste foi pouco utilizado, visto que não existem relatos sobre padronização ou pesquisas com o mesmo dentro da literatura consultada $^{(6)}$. Em 1996, um consenso entre pesquisadores norte-americanos sugeriu a inclusão de testes que avaliassem os aspectos temporais da fala, tais como: resolução, integração, ordenação e sequencialização, na avaliação do processamento auditivo $^{(7)}$ e houve o renascimento do interesse por testes capazes de avaliar a resolução temporal. O teste WAFT foi então revisado e deu origem ao Auditory Fusion Test-Revised - Teste de Fusão Auditiva Revisado (AFT-R ${ }^{(4,6)}$. O AFT-R foi padronizado em sujeitos norte-americanos com idades entre três e 70 anos. Os resultados mostraram melhora progressiva do limiar de fusão auditiva até nove anos de idade, estabilizados até a idade adulta e decréscimo a partir da terceira idade. Sujeitos com queixas de dificuldade na linguagem oral e escrita apresentaram limiares de fusão auditiva aumentados; desta forma, o AFT-R mostrou-se eficiente na detecção de transtornos da habilidade de resolução temporal auditiva ${ }^{(6)}$.

Em 2000, após alguns experimentos utilizando o AFT-R, verificou-se que esse teste necessitava de uma nova revisão para que sua aplicação fosse mais rápida e para eliminar a probabilidade de predição das respostas. Foi desenvolvido, então, o Random Gap Detection Test - Teste de Detecção de Intervalo Aleatório (RGDT). A utilização do AFT-R já havia demonstrado que indivíduos sem transtorno da habilidade de resolução temporal apresentavam limiar de fusão auditiva abaixo de $40 \mathrm{~ms}$. Assim, o RGDT foi desenvolvido com apresentações, cujos intervalos não ultrapassam 40 ms. No AFT-R, a apresentação dos intervalos era feita em ordem crescente e decrescente e possibilitava ao examinado a predição das respostas e, ao mesmo tempo, impossibilitava o examinador distinguir entre as respostas corretas e as inferidas. Por esse motivo, os intervalos apresentados no RGDT foram padronizados em ordem aleatória. O teste consiste na apresentação de tons puros pareados com pequenos intervalos de silêncio, que variam de zero a $40 \mathrm{~ms}$ apresentados em ordem aleatória. O sujeito é orientado a responder verbalmente ou apontar se ouviu um ou dois estímulos. O limiar de detecção de intervalo é considerado como sendo o menor intervalo a partir do qual o indivíduo passou a identificar consistentemente a ocorrência de dois estímulos. Espera-se que indivíduos sem alterações da resolução temporal auditiva consigam identificar intervalos de silêncio até $20 \mathrm{~ms}$. O RGDT mostrou-se sensível a transtornos da resolução temporal auditiva que, segundo o autor, podem estar relacionadas a transtornos do processamento fonológico, problemas de discriminação auditiva, linguagem receptiva e leitura. Para tais apontamentos, o autor do teste cita como exemplo a diferença no tempo de duração entre os fonemas $/ \mathrm{p} /$ e $/ \mathrm{b} /$, que é de aproximadamente $20 \mathrm{~ms}$, logo, se o limiar de resolução temporal de um indivíduo for superior a $20 \mathrm{~ms}$, o mesmo pode apresentar dificuldade para discriminar os sons da fala. O RGDT é um teste que avalia a habilidade de resolução temporal, identificando e qualificando os transtornos de timing do sistema auditivo ${ }^{(4)}$.

A identificação da ordem para pares de itens, como por exemplo, a discriminação entre palavras similares, tais como: gato e gasto, pote e poste, dentre outras, requer resolução temporal íntegra. Se o limiar de resolução temporal do sujeito for maior do que a duração dos fonemas que compõem a palavra ouvida, este sujeito terá dificuldade para discriminar traços acústicos de fala mesmo que conheça a língua e faça uso de sua redundância extrínseca ${ }^{(8)}$. Quando apresentamos pares de ruídos em sucessões rápidas, separadas por uma pausa ou intervalo muito pequeno e variável, indivíduos jovens normais detectam intervalos de tempo da ordem de 2 a $3 \mathrm{~ms}$. Entretanto, um intervalo em torno de $20 \mathrm{~ms}$ é necessário para que o mesmo sujeito possa indicar a ordem desses mesmos estímulos ${ }^{(9)}$.

Tendo em vista a importância da habilidade de resolução temporal para o processamento do sinal de fala, o presente estudo teve por objetivo verificar o efeito do envelhecimento na habilidade de resolução temporal auditiva por meio do Teste de Detecção de Intervalo Aleatório - Random Gap Detection Test (RGDT).

\section{MÉTODOS}

Trata-se de uma pesquisa exploratória, descritiva e quantitativa. Os dados somente foram coletados após a aprovação do projeto pelo Comitê de Ética em Pesquisa do Centro de Especialização em Fonoaudiologia Clínica (CEFAC), sob número 80/06. De acordo com as normas preconizadas para experiências com seres humanos, todos os participantes assinaram o Termo de Consentimento Livre e Esclarecido. A coleta de dados foi realizada em uma clínica particular, localizada na Zona Norte do município de São Paulo.

Participaram deste estudo 72 mulheres, distribuídas em dois grupos: grupo controle (GC), constituído por 48 jovens 
com idades entre 20 e 39 anos (média: 23,8 anos, DP: \pm 4 anos, mediana: 22 anos); e grupo experimental (GE), constituído por 24 idosas, com idades entre 60 e 75 anos (média: 66,8 anos, DP: $\pm 4,5$ anos, mediana: 67 anos).

Os indivíduos que compunham GC apresentavam limiares de audição por via aérea nas frequiências de 250, 500, 1000, 2000, 3000, 4000, 6000 e $8000 \mathrm{~Hz}$ dentro dos padrões de normalidade, ou seja, até $25 \mathrm{~dB}$ NA, curva timpanométrica do tipo A bilateralmente, sugerindo normalidade da função da orelha média e ausência de queixas auditivas atuais ou prévias.

O GE foi constituído pelos indivíduos que obedeceram aos seguintes critérios de inclusão: limiares de audição por via aérea, nas frequências de 250, 500, 1000, 2000, 3000, 4000, 6000 e $8000 \mathrm{~Hz}$ dentro dos padrões de normalidade ou perda do tipo neurossensorial de grau leve, ou seja, média dos limiares de 500,1000 e $2000 \mathrm{~Hz}$ até $40 \mathrm{~dB}$ NA com configuração audiométrica horizontal ou descendente; curva timpanométrica do tipo A bilateralmente, sugerindo normalidade da função da orelha média e ausência de queixas auditivas atuais ou prévias.

Por conta das diferenças na configuração audiométrica dos sujeitos que compunham o GE, este grupo foi distribuído em três subgrupos: GE-I, composto por três idosas que apresentavam limiares audiométricos até $25 \mathrm{~dB}$ NA de 250 a $8000 \mathrm{~Hz}$; GE-II, composto por 15 idosas que apresentavam média dos limiares entre 500, 1000 e $2000 \mathrm{~Hz}$ até $25 \mathrm{~dB}$ NA e configuração descendente (perda auditiva nas frequiências altas); e, GE-III, composto por seis idosas que apresentavam média dos limiares entre 500, 1000 e $2000 \mathrm{~Hz}$ entre 26 e $40 \mathrm{~dB}$ NA (perda auditiva leve). Cabe ressaltar que os resultados não foram separados por orelha, pois foram incluídos nesta pesquisa somente os sujeitos que apresentavam audição simétrica, ou seja, curva audiométrica de mesmo grau e mesma configuração em ambas as orelhas.

Os sujeitos foram submetidos ao RGDT para tom puro, nas frequências de 500,1000, 2000 e $4000 \mathrm{~Hz}$, sendo os estímulos apresentados a $50 \mathrm{~dB}$ NS, na condição binaural, conforme protocolo do teste. Cada frequência é composta por nove apresentações de tons puros pareados, cujo tempo de intervalo entre os tons varia de zero a $40 \mathrm{~ms}$ em ordem aleatória, com incrementos que variam de dois a $10 \mathrm{~ms}$. Os indivíduos foram orientados, a cada apresentação, a apontar se estavam ouvindo um ou dois tons.

$\mathrm{Na}$ análise do RGDT, foi considerado o menor intervalo a partir do qual o indivíduo passou a identificar a presença de dois tons consistentemente. Inicialmente obtivemos o valor do menor intervalo por frequência e, em seguida, obtivemos a média entre as quatro frequências avaliadas.

As variáveis quantitativas obtidas no GC foram submetidas a testes de média, desvio padrão, mediana e amplitude interquartílica. Para a análise estatística, inicialmente verificamos possíveis influências da idade no desempenho do RGDT. Para o GC não foi necessário fazer qualquer subdivisão, visto que os resultados são uma constante; logo, o efeito da variável idade sobre os resultados desse grupo não existem. Para o GE foi realizado o teste não paramétrico de Mann-Whitney com o objetivo de comparar as faixas etárias de 60 a 69 anos e 70 a 79 anos para cada frequiência de teste. Posteriormente, desconsiderada a segregação da amostra em faixas etárias para ambos os grupos, o GC foi mantido em um único grupo e o GE foi dividido de acordo com a configuração audiométrica, conforme já discutido anteriormente. A variável qualitativa "identificar ou não o intervalo de silêncio até $40 \mathrm{~ms}$ ", comparando os resultados de GC e do GE também foram submetidas ao teste não paramétrico de Mann-Whitney. Para todas as significâncias calculadas (p), foram adotados os valores abaixo de $5 \%$, ou seja, $\mathrm{p} \leq 0,050$, como valor de significância.

\section{RESULTADOS}

Na Tabela 1 encontra-se a distribuição dos sujeitos do GC e do GE que identificaram ou não o intervalo até $40 \mathrm{~ms}$ em cada freqüência avaliada no RGDT. No GE observamos que 20 dos 24 indivíduos não identificaram o intervalo até $40 \mathrm{~ms}$ em uma ou mais frequências.

Tabela 1. Número de sujeitos do GC e do GE que identificaram ou não o intervalo até $40 \mathrm{~ms}$ no RGDT, de acordo com a frequência de teste

\begin{tabular}{|c|c|c|c|c|}
\hline \multirow[t]{2}{*}{$\begin{array}{l}\text { Frequência } \\
(\mathrm{Hz})\end{array}$} & \multicolumn{2}{|c|}{$\begin{array}{c}\text { Identificaram o } \\
\text { intervalo até } 40 \mathrm{~ms}\end{array}$} & \multicolumn{2}{|c|}{$\begin{array}{l}\text { Não identificaram o } \\
\text { intervalo até } 40 \mathrm{~ms}\end{array}$} \\
\hline & $\mathrm{GC}(\mathrm{N}=48)$ & GE $(N=24)$ & $\mathrm{GC}(\mathrm{N}=48)$ & $\mathrm{GE}(\mathrm{N}=24)$ \\
\hline 500 & 48 & 5 & 0 & 19 \\
\hline 1000 & 48 & 9 & 0 & 15 \\
\hline 2000 & 48 & 8 & 0 & 16 \\
\hline 4000 & 48 & 9 & 0 & 15 \\
\hline Significância (p) & \multicolumn{2}{|c|}{$<0,001$} & \multicolumn{2}{|c|}{$<0,001$} \\
\hline
\end{tabular}

Legenda: $\mathrm{GC}=$ grupo controle (sujeitos jovens); $\mathrm{GE}$ = grupo experimental (sujeitos idosos)

Na Tabela 2 verifica-se os resultados dos limiares do RGDT para o GC, em milissegundos, por frequência e a média entre as quatro frequências avaliadas. O intervalo médio de respostas entre as frequências avaliadas no GC foi de 12,2 ms (DP \pm 5 ).

Tabela 2. Resultados do RGDT, em milissegundos, por frequência e média entre as quatro frequências avaliadas, obtidos para o $\mathrm{GC}(\mathrm{N}=48)$

\begin{tabular}{|c|c|c|c|c|c|}
\hline & $500 \mathrm{~Hz}$ & $1000 \mathrm{~Hz}$ & $2000 \mathrm{~Hz}$ & $4000 \mathrm{~Hz}$ & $\begin{array}{c}\text { Média de } \\
500 \text { a } 4000 \\
\mathrm{~Hz}\end{array}$ \\
\hline Média & 14,6 & 12,4 & 10,7 & 11 & 12,2 \\
\hline Desvio Padrão & 8,6 & 7,1 & 5,1 & 5,3 & 5 \\
\hline Mediana & 15 & 10 & 10 & 10 & 11,3 \\
\hline Mínimo & 2 & 2 & 2 & 2 & 4 \\
\hline Máximo & 40 & 40 & 25 & 30 & 26,3 \\
\hline Percentil 25 & 10 & 8,8 & 8,8 & 10 & 8,8 \\
\hline Percentil 75 & 20 & 15 & 15 & 15 & 15 \\
\hline
\end{tabular}

A Tabela 3 refere-se à análise estatística do número de sujeitos do GE que identificaram ou não o intervalo até 40 ms em cada frequência avaliada de acordo com a faixa etária. Verificamos que não houve efeito da idade para nenhuma das frequências de teste.

A Tabela 4 representa o número de sujeitos do GE que identificaram ou não o intervalo até $40 \mathrm{~ms}$ em cada frequência 
Tabela 3. Número de sujeitos do GE que identificaram ou não o intervalo até $40 \mathrm{~ms}$ no RGDT, por frequência, de acordo com faixa etária

\begin{tabular}{lccccc}
\hline Faixa etária & \multicolumn{2}{c}{$60-69(\mathrm{~N}=19)$} & \multicolumn{2}{c}{ S0-79 $(\mathrm{N}=5)$} & \multicolumn{2}{c}{ Significância } \\
\cline { 1 - 5 } Frequência $(\mathrm{Hz})$ & Identificaram & Não identificaram & Identificaram & Não identificaram & \\
\hline 500 & 4 & 15 & 1 & 4 & $\mathrm{p}=0,960$ \\
1000 & 6 & 13 & 3 & 3 & $\mathrm{p}=0,253$ \\
2000 & 6 & 13 & 2 & 2 & $\mathrm{p}=0,728$ \\
4000 & 6 & 13 & 3 & $\mathrm{p}=0,253$ \\
\hline
\end{tabular}

Tabela 4. Número de sujeitos do GE que identificaram ou não o intervalo até 40 ms no RGDT, por frequência, de acordo com a configuração audiométrica

\begin{tabular}{lcccccc}
\hline Frequência $(\mathrm{Hz})$ & \multicolumn{2}{c}{$\mathrm{GE}-\mathrm{I}(\mathrm{N}=3)$} & \multicolumn{2}{c}{ GE-II $(\mathrm{N}=15)$} & \multicolumn{2}{c}{$\mathrm{GE}-\mathrm{III}(\mathrm{N}=6)$} \\
\cline { 2 - 7 } & Identificaram & Não identificaram & Identificaram & Não identificaram & Identificaram & Não identificaram \\
\hline 500 & 0 & 3 & 5 & 10 & 0 & 6 \\
1000 & 0 & 3 & 9 & 6 & 0 & 6 \\
2000 & 1 & 2 & 7 & 8 & 0 & 6 \\
4000 & 0 & 3 & 9 & 6 & 6 & 6 \\
\hline
\end{tabular}

Legenda: GE-I = grupo experimental I (limiares audiométricos dentro da normalidade e configuração horizontal); GE-II = grupo experimental II (média tonal dentro da normalidade e configuração descendente); GE-III = grupo experimental III (média tonal de grau leve e configuração descendente)

avaliada de acordo com a configuração audiométrica. Nessa análise também encontramos um número significativo de idosas que não identificaram o intervalo até $40 \mathrm{~ms}$ em uma ou mais frequências avaliadas independente da configuração audiométrica.

\section{DISCUSSÃO}

A análise dos dados da Tabela 1 mostrou diferença estatisticamente significante entre os dois grupos com $\mathrm{p}<0,001$ nas quatro frequências avaliadas no RGDT. Esses resultados concordam com a literatura pesquisada. Um estudo com grupo de 30 idosos submetidos ao AFT-R observou que dez idosos da amostra não conseguiram identificar o intervalo de até $60 \mathrm{~ms}^{(2)}$.

O RGDT foi padronizado em crianças entre cinco e 11 anos e o valor de normalidade estabelecido para esta população foi de até $20 \mathrm{~ms}$ para a média dos resultados entre 500 e $4000 \mathrm{~Hz}^{(4)}$. Na Tabela 2 observou-se que o intervalo médio de respostas entre as frequências avaliadas no GC foi de 12,2 ms (DP \pm 5 ). $\mathrm{O}$ valor médio no AFT-R, padronizado em adultos norte-americanos é de $8 \mathrm{~ms}(\mathrm{DP} \pm 3)^{(6)}$. Esta análise foi realizada apenas no GC, pois, conforme mostra a Tabela 1, a maioria dos sujeitos do GE não conseguiu identificar o intervalo até $40 \mathrm{~ms}$.

Os resultados apresentados nas Tabelas 3 e 4 também vão ao encontro da literatura pesquisada. Autores apontaram que a idade e a perda auditiva contribuem de forma independente para os transtornos do processamento temporal. Autores demonstraram que: (1) o aumento da idade pode ocasionar diminuição da eficiência dos mecanismos centrais de timing, (2) a deterioração na habilidade de resolução temporal sofre interferência da idade independente da presença de perda auditiva periférica, e (3) as dificuldades de reconhecimento de fala em idosos podem ser consequência do declínio da sensibilidade temporal. Pesquisadores acreditam que os efeitos da idade podem justificar a limitação na habilidade de processar rapidamente os segmentos da fala e sugerem que, independente do indivíduo ser jovem ou idoso, a presença da perda auditiva não afeta o desempenho dos testes ${ }^{(10-21)}$.

Alterações decorrentes do envelhecimento no processamento auditivo ocorrem por toda terceira idade e, especificamente, mudanças relacionadas à idade na acuidade temporal podem começar antes das mudanças no limiar auditivo ou no reconhecimento de palavras ${ }^{(22)}$, o que explicaria algumas queixas para compreender a fala e dificuldades de comunicação mesmo sem a presença de perda auditiva periférica ${ }^{(1-2)}$.

Sendo a integridade dos aspectos temporais da audição prérequisito para que o sistema auditivo execute corretamente o processamento fonológico e a discriminação auditiva de pistas temporais da fala, idosos que referem ouvir, mas não entender podem apresentar a habilidade de resolução temporal deteriorada pelo envelhecimento ${ }^{(3-5)}$. Sujeitos idosos que apresentam limiar de resolução temporal maior do que a duração dos fonemas que compõem a palavra ouvida, ou seja, limiar de resolução temporal superior a $20 \mathrm{~ms}$, podem apresentar dificuldades para discriminar palavras auditivamente similares, mesmo conhecendo a língua e fazendo uso de sua redundância extrínseca ${ }^{(4-8)}$.

Se indivíduos jovens normais detectam intervalos de tempo da ordem de 2 a $3 \mathrm{~ms}^{(9)}$, a presente pesquisa sugere que o sujeito idoso parece necessitar de um intervalo maior para identificar a presença de dois tons independente da audição periférica; caso contrário, os sujeitos do GE-I, que possuíam audição periférica normal, apresentariam limiares de resolução temporal semelhantes aos jovens.

Acredita-se que o avanço da idade pode ser um fator significativo nos transtornos da resolução temporal. No presente estudo, o RGDT mostrou ser um importante instrumento na avaliação da integridade funcional do processamento temporal auditivo em idosos, visto que essa população apresentou resolução temporal pior, em milissegundos, quando comparada à população adulta jovem para intervalos de curta duração. Por esta razão, acredita-se que futuras pesquisas devam ser realizadas com testes que disponham de intervalos aleatórios 
superiores a $40 \mathrm{~ms}$ para que esta população possa detectar a presença destes intervalos, determinando assim o limiar de resolução temporal dos idosos.

\section{CONCLUSÃO}

A partir dos resultados, concluímos que um número es- tatisticamente significante de idosos (20:24) não detectou o intervalo até $40 \mathrm{~ms}$ em uma ou mais das quatro frequências avaliadas; o avanço da idade mostrou ser fator de piora no desempenho da habilidade de resolução temporal; o RGDT mostrou ser um instrumento adequado para avaliar a evolução dos efeitos do envelhecimento na habilidade de resolução temporal auditiva.

\begin{abstract}
Purpose: To compare the performances of young and elderly women on the Random Gap Detection Test (RGDT). Methods: Seventy two women were evaluated, distributed in two groups: Control Group (CG), composed by 48 young women (mean age $=23.8$ years) with normal peripheral hearing, and Experimental Group (EG), composed by 24 elderly women (mean age $=66.8$ years) with normal peripheral hearing or mild sensorineural hearing loss. Both groups were submitted to the RGDT for pure-tones, in the frequencies of 500, 1000, 2000 and $4000 \mathrm{~Hz}$. Results: All subjects from CG identified gaps up to $40 \mathrm{~ms}$ in all tested frequencies, with average gap of $12.2 \mathrm{~ms}$ among them. In the EG, it was observed that 20 out of the 24 subjects did not identify intervals up to $40 \mathrm{~ms}$ in one or more frequencies. Data analysis showed statistically significant differences between the performances of the groups $(\mathrm{p}<0001)$. Conclusions: Age increase was shown as an aggravating factor for performance on the RGDT. This test proved to be a valuable tool for the evaluation of aging effects on auditory temporal resolution ability.
\end{abstract}

Keywords: Aged; Aging; Hearing; Hearing disorders; Auditory perception; Auditory perceptual disorders; Hearing tests; Auditory diseases, central

\title{
REFERÊNCIAS
}

1. Neves VT, Feitosa MAG. Controvérsias ou complexidade na relação entre processamento temporal auditivo e envelhecimento? Rev Bras Otorrinolaringol. 2003;69(2):242-9.

2. Rose NV, Feniman MR. Desempenho de idosos no teste de fusão auditiva- revisado (AFT-R) - gap detection. J Bras Fonoaudiol. 2001;2(6):71-4.

3. Durrant JD, Lovrinic JH. Basis of hearing science. 3rd ed. Baltimore: Willians \& Wilkins; 1995. p. 299.

4. Keith RW. RGDT - Random gap detection test. Auditec of St. Louis; 2000.

5. Balen SA. Processamento auditivo central: aspectos temporais da audição e percepção acústica da fala. [dissertação]. São Paulo: Pontifícia Universidade Católica de São Paulo; 1997.

6. Davis SM, McCroskey RL. Auditory fusion in children. Child Dev. 1980;51(1):75-80.

7. American Speech-Language-Hearing Association (ASHA). Task force on central auditory processing consensus development. Central auditory processing: current status of research and implications for clinical practice. Technical report; 1996.

8. Warren RM. Perception of acoustic sequences: global integration versus temporal resolution. In: McAdams S, Bigand E, editors. Thinking in sound: the cognitive psychology of human audition. New York: Oxford University Press; 2001. p. 37, 42.

9. Gelfand SA. Measurement principles and the nature of hearing. In: Gelfand SA. Essentials of audiology. New York: Thieme; 1997. p. 99

10. Fitzgibbons PJ, Gordon-Salant S. Age effects on measures of auditory duration discrimination. J Speech Hear Res. 1994;37(3):662-70.

11. Fitzgibbons PJ, Gordon-Salant S. Age effects on duration discrimination with simple and complex stimuli. J Acoust Soc Am. 1995;98(6):3140-5.
12. Fitzgibbons PJ, Gordon-Salant S. Auditory temporal order perception in younger and older adults. J Speech Lang Hear Res. 1998;41(5):1052-60.

13. Fitzgibbons PJ, Gordon-Salant S. Aging and temporal discrimination in auditory sequences. J Acoust Soc Am. 2001;109(6):2955-63.

14. Fitzgibbons PJ, Gordon-Salant S. Age effects on discrimination of timing in auditory sequences. J Acoust Soc Am. 2004;116(2):1126-34.

15. Fitzgibbons PJ, Gordon-Salant S, Friedman SA. Effects of age and sequence presentation rate on temporal order recognition. J Acoust Soc Am. 2006;120(2):991-9.

16. Fitzgibbons PJ, Gordon-Salant S, Barrett J. Age-related differences in discrimination of an interval separating onsets of successive tone bursts as a function 17. Gordon-Salant S. Hearing loss and aging: new research findings and clinical implications. J Rehabil Res Dev. 2005;42(4 Suppl 2):9-24.

18. Gordon-Salant S, Fitzgibbons PJ. Temporal factors and speech recognition performance in young and elderly listeners. J Speech Hear Res. 1993;36(6):1276-85.

19. Gordon-Salant S, Fitzgibbons PJ. Profile of auditory temporal processing in older listeners. J Speech Lang Hear Res. 1999;42(2): 30011.

20. Gordon-Salant S, Fitzgibbons PJ. Effects of stimulus and noise rate variability on speech perception by younger and older adults. J Acoust Soc Am. 2004;115(4): 1808-17.

21. Gordon-Salant S, Yeni-Komshian GH, Fitzgibbons PJ, Barrett J. Agerelated differences in identification and discrimination of temporal cues in speech segments. J Acoust Soc Am. 2006;119(4):2455-66.

22. Snell KB, Frisina DR. Relationships among age-related differences in gap detection and word recognition. J Acoust Soc Am. 2000;107(3):1615-26. 